\title{
Lyapunov Conditions for Input-to-State Stability of Impulsive Systems *
}

\author{
João P. Hespanha ${ }^{\mathrm{a}, 1}$, Daniel Liberzon ${ }^{\mathrm{b}}, \quad$ Andrew R. Teel ${ }^{\mathrm{a}}$ \\ ${ }^{\mathrm{a}}$ University of California, Santa Barbara, CA 93106-9560, U.S.A. \\ ${ }^{\mathrm{b}}$ University of Illinois at Urbana-Champaign, Urbana, IL 61801, U.S.A.
}

\begin{abstract}
This paper introduces appropriate concepts of input-to-state stability (ISS) and integral-ISS for impulsive systems, i.e., dynamical systems that evolve according to ordinary differential equations most of the time, but occasionally exhibit discontinuities (or impulses). We provide a set of Lyapunov-based sufficient conditions for establishing these ISS properties. When the continuous dynamics are ISS but the discrete dynamics that govern the impulses are not, the impulses should not occur too frequently, which is formalized in terms of an average dwell-time (ADT) condition. Conversely, when the impulse dynamics are ISS but the continuous dynamics are not, there must not be overly long intervals between impulses, which is formalized in terms of a novel reverse ADT condition. We also investigate the cases where (i) both the continuous and discrete dynamics are ISS and (ii) one of these is ISS and the other only marginally stable for the zero input, while sharing a common Lyapunov function. In the former case we obtain a stronger notion of ISS, for which a necessary and sufficient Lyapunov characterization is available. The use of the tools developed herein is illustrated through examples from a Micro-Electro-Mechanical System (MEMS) oscillator and a problem of remote estimation over a communication network.
\end{abstract}

Key words: Impulsive systems; Input-to-state stability; Nonlinear systems

\section{Introduction}

Impulsive systems combine continuous evolution (typically modeled by ordinary differential equations) with instantaneous state jumps or resets (also referred to as impulses). Stability properties of such systems have been extensively investigated in the literature; see, e.g., $[3,7,25]$.

When investigating stability of a system, it is important to characterize the effects of external inputs. The concepts of input-to-state stability (ISS) and integral-inputto-state stability (iISS), introduced by Sontag in [23]

\footnotetext{
* J. Hespanha supported by the NSF grant No. CNS0720842 and the ARO grant DAAD19-03-1-0144. A. Teel supported by the grant NSF ECS-0622253 and the AFOSR grant F9550-06-1-0134. D. Liberzon supported by the NSF grant ECS-0134115 CAR and the DARPA/AFOSR MURI grant F49620-02-1-0325.

Email addresses: hespanha@ece.ucsb.edu (João P. Hespanha), liberzon@uiuc.edu (Daniel Liberzon), teel@ece.ucsb.edu (Andrew R. Teel).

1 Corresponding author. Email: hespanha@ece.ucsb.edu, Phone: +1 (805) 893-7042, Fax: +1 (805) 893-3262
}

and [22], have proved useful in this regard. Originally introduced for continuous-time systems, they were subsequently also studied for discrete-time systems [12] and switched systems [17]. However, the possibility of impulses has been excluded in these works. ISS notions for hybrid systems appeared in [4]. While [4] allows for the existence of impulses, in [4] signals are defined on hybrid time domains, as opposed to the usual time defined on the real line. This leads to a distinct notion of ISS and some systems that are ISS in the framework of this paper are not ISS in the framework of [4]. This issue is further discussed in Section 5.

In this paper we study input-to-state stability properties of impulsive systems, with external signals affecting both the continuous dynamics and the state impulse map. These systems are formally defined in Section 2, where we also define the notions of ISS and iISS for such systems.

We provide a set of Lyapunov-based sufficient conditions for establishing ISS and iISS with respect to suitable classes of impulse time sequences (see Sections 3 and 4 for ISS, and Section 7 for iISS). It is shown that when the continuous dynamics are ISS but the impulses 
are destabilizing, the impulsive system is ISS if the impulse times do not occur too frequently, which is formalized in terms of an average dwell-time (ADT) condition from [9]. Conversely, when the impulses are stabilizing but the continuous dynamics are destabilizing, the impulsive system is ISS if the impulse times satisfy a novel "reverse" ADT condition, which prevents overly long intervals between impulse times.

Section 5 considers impulsive systems for which both the continuous dynamics and the impulses are stabilizing, and share a common ISS-Lyapunov function. Such systems are ISS regardless of how often or how seldom impulses occur. We further show that such systems exhibit a stronger form of ISS for which we provide necessary and sufficient conditions in terms of the existence of appropriate Lyapunov functions.

We also investigate impulsive systems for which the continuous dynamics are ISS and the impulse dynamics are marginally stable for a zero input. We show that such systems remain ISS for arbitrarily small ADT. We also consider the dual case, which consists of systems with ISS impulse dynamics and continuous dynamics that are marginally stable for a zero input. These systems remain ISS for arbitrarily large reverse ADT. Lyapunov-based conditions that cover both cases are provided in Section 6 .

The motivation to study the class of systems considered in this paper comes from multiple sources. Impulsive systems with external inputs arise naturally in control systems with communication constraints, as explicitly discussed in $[10,16,19,28]$. A special case of one of our results (Corollary 3) was already used in [16] to analyze stability of such a system. The results presented here can be used to construct deterministic versions of the results that appeared in [28] for stochastic disturbances. Impulsive systems with inputs also describe the evolution of multiple Lyapunov functions for switched systems with inputs (even if the latter exhibit no state jumps), which in turn arise in the analysis of switching control algorithms for uncertain systems [8, 15].

We illustrate the use of the results presented in this paper through two examples included in Section 8. The first example studies the effect of the collision of air molecules with a MEMS oscillator. These collisions can be the main source of noise in mass-sensing applications. The second example studies the effect of a TOD protocol in the state estimation of multiple decoupled systems that share the same communication medium to transmit measurements to a remote location where the state estimates are being built.

\section{Basic definitions}

We consider the general impulsive system with inputs

$$
\begin{cases}\dot{x}(t)=f(x(t), w(t)), & t \neq t_{k}, k \in\{1,2, \ldots\} \\ x(t)=g\left(x^{-}(t), w^{-}(t)\right), & t=t_{k}, k \in\{1,2, \ldots\}\end{cases}
$$

where $\left\{t_{1}, t_{2}, t_{3}, \ldots\right\}$ is a strictly increasing sequence of impulse times in $\left(t_{0}, \infty\right)$ for some initial time $t_{0}$; the state $x(t) \in \mathbb{R}^{n}$ is absolutely continuous between impulses; $w(t) \in \mathbb{R}^{m}$ is a locally bounded, Lebesgue-measurable input; and $f$ and $g$ are functions from $\mathbb{R}^{n} \times \mathbb{R}^{m}$ to $\mathbb{R}^{n}$, with $f$ locally Lipschitz. The set of impulse times is assumed to be either finite or infinite and unbounded. In particular, we exclude the possibility of the $t_{k}$ having a finite accumulation point, often referred to as chattering. All signals in this paper (including the state $x$ and the input $w$ ) are assumed to be right-continuous and to have left limits at all times ${ }^{2}$. In view of this, we denote by $(\cdot)^{-}$the left-limit operator, i.e., $x^{-}(t)=\lim _{s}{ }_{t} x(s)$. Given a sequence $\left\{t_{k}\right\}$ and a pair of times $s, t$ satisfying $t>s \geq t_{0}$, we will let $N(t, s)$ denote the number of impulse times $t_{k}$ in the semi-open interval $(s, t]$.

To introduce appropriate notions of ISS, we recall the following standard definitions: A function $\alpha:[0, \infty) \rightarrow$ $[0, \infty)$ is of class $\mathcal{K}$, and we write $\alpha \in \mathcal{K}$, when $\alpha$ is continuous, strictly increasing, and $\alpha(0)=0$. If $\alpha$ is also unbounded, then we say it is of class $\mathcal{K}_{\infty}$ and write $\alpha \in \mathcal{K}_{\infty}$. A function $\beta:[0, \infty) \times[0, \infty) \rightarrow[0, \infty)$ is of class $\mathcal{K} \mathcal{L}$, and we write $\beta \in \mathcal{K} \mathcal{L}$, when $\beta(\cdot, t)$ is of class $\mathcal{K}$ for each fixed $t \geq 0$ and $\beta(r, t)$ decreases to 0 as $t \rightarrow \infty$ for each fixed $r \geq 0$.

Suppose that a sequence $\left\{t_{k}\right\}$ is given. We say that the impulsive system (1) is input-to-state stable (ISS) if there exist functions $\beta \in \mathcal{K} \mathcal{L}$ and $\gamma \in \mathcal{K}_{\infty}$ such that for every initial condition and every input $w$, the corresponding solution to (1) exists globally and satisfies

$$
|x(t)| \leq \beta\left(\left|x\left(t_{0}\right)\right|, t-t_{0}\right)+\gamma\left(\|w\|_{\left[t_{0}, t\right]}\right) \quad \forall t \geq t_{0}
$$

where $\|\cdot\|_{J}$ denotes the supremum norm on an interval $J$.

Since the above definition applies to a fixed sequence $\left\{t_{k}\right\}$ of impulse times, the ISS property depends on the choice of the sequence. However, it is often of interest to characterize ISS over classes of sequences $\left\{t_{k}\right\}$. To this end, we say that the impulsive system (1) is uniformly $I S S$ over a given class $\mathcal{S}$ of admissible sequences of impulse times if the ISS property expressed by (2) holds for every sequence in $\mathcal{S}$ with functions $\beta$ and $\gamma$ that are independent of the choice of the sequence.

\footnotetext{
${ }^{2}$ Right-continuity of $w$ is being assumed just for simplicity of notation and it is not necessary for the results to hold.
} 
The above ISS properties characterize robustness to inputs in the $L_{\infty}$ sense. Another possibility is to consider "integral" variants, in the spirit of [22]. We say that the impulsive system (1) is integral-input-to-state stable (iISS) if there exist functions $\beta \in \mathcal{K} \mathcal{L}$ and $\alpha, \gamma \in \mathcal{K}_{\infty}$ such that for every initial condition and every input $w$, we have

$$
\begin{gathered}
\alpha(|x(t)|) \leq \beta\left(\left|x\left(t_{0}\right)\right|, t-t_{0}\right)+\int_{t_{0}}^{t} \gamma(|w(s)|) d s \\
+\sum_{t_{k} \in\left[t_{0}, t\right]} \gamma\left(\left|w^{-}\left(t_{k}\right)\right|\right) \quad \forall t \geq t_{0} .
\end{gathered}
$$

The notion of uniform iISS over a given class $\mathcal{S}$ of impulse time sequences is defined in the same way as for ISS.

\section{Sufficient conditions for ISS}

We say that a function $V: \mathbb{R}^{n} \rightarrow \mathbb{R}$ is a candidate exponential ISS-Lyapunov function for (1) with rate coefficients $c, d \in \mathbb{R}$ if $V$ is locally Lipschitz, positive definite, radially unbounded, and satisfies

$$
\begin{gathered}
\nabla V(x) \cdot f(x, w) \leq-c V(x)+\chi(|w|) \quad \forall x \text { a.e., } \forall w \\
V(g(x, w)) \leq e^{-d} V(x)+\chi(|w|) \quad \forall x, w \quad
\end{gathered}
$$

for some function ${ }^{3} \chi \in \mathcal{K}_{\infty}$. In (4a) and in equations that follow, " $\forall x$ a.e." should be interpreted as "for every $x \in \mathbb{R}^{n}$ except, possibly, on a set of zero Lebesguemeasure in $\mathbb{R}^{n}$." For generality, we are assuming that $V$ is locally Lipschitz but not necessarily differentiable everywhere. However, from Rademacher's Theorem we know that the former is sufficient to guarantee that the gradient $\nabla V(x)$ of $V(x)$ is well defined except on a set of measure zero. For this reason we qualify the $x$ quantifier in (4a) with "almost everywhere."

We do not require the rate coefficients $c, d$ to be nonnegative and therefore $V$ will not necessarily decrease, even when $w=0$. The next result says that when these coefficients satisfy appropriate constraints, one can still use $V$ to show that the impulsive system is ISS.

Theorem 1 (uniform ISS) Let $V$ be a candidate exponential ISS-Lyapunov function for (1) with rate coefficients $c, d \in \mathbb{R}$ with $d \neq 0^{4}$. For arbitrary constants

\footnotetext{
3 Taking the same function $\chi$ in $(4 a)$ and $(4 b)$ is no loss of generality, because we can always consider the maximum of two functions; however, it is also easy to treat the case of two different functions, which would lead to slightly more complicated notation but less conservative estimates for the gain function $\gamma$ in (2).

${ }^{4}$ The case $d=0$ is closely related to the results in Section 6 .
}

$\mu, \lambda>0$, let $\mathcal{S}[\mu, \lambda]$ denote the class of impulse time sequences $\left\{t_{k}\right\}$ satisfying

$$
-d N(t, s)-(c-\lambda)(t-s) \leq \mu \quad \forall t \geq s \geq t_{0} .
$$

Then the system (1) is uniformly ISS over $\mathcal{S}[\mu, \lambda]$.

After proving Theorem 1, we will provide additional insight into the somewhat mysterious condition (5). When none of the rate coefficients $c$ and $d$ is positive, this condition cannot hold for any impulse time sequence because the left-hand side will necessarily grow to $\infty$ as $t-s \rightarrow \infty$. All other combinations of signs for $c$ and $d$ lead to interesting results. Section 4 explores the case when one coefficient is strictly positive and the other strictly negative, in which case we have uniform ISS for impulse sequences that satisfy appropriate "dwell-time" conditions. Section 5 addresses the case when both coefficients are strictly positive. In this case, (5) always holds and the system actually exhibits a form of uniform ISS that is stronger than the one that appears in Theorem 1. Finally, Section 6 addresses the marginal cases when one coefficient is strictly positive and the other one is zero.

Proof of Theorem 1. Pick constants $\varepsilon>-1$, of the same sign as $d$, and $\delta>0$, both sufficiently close to 0 so that $d>\bar{d}:=\frac{d}{1+\varepsilon}, c>\bar{c}:=\frac{c-\delta}{1+\varepsilon}, \bar{\lambda}:=\frac{\lambda-\delta}{1+\varepsilon}>0$. Adding $\delta(t-s)$ to both sides of (5) and then dividing both sides by $1+\varepsilon$, we conclude that

$$
-\bar{d} N(t, s)-\bar{c}(t-s) \leq \bar{\mu}-\bar{\lambda}(t-s) \quad \forall t \geq s \geq t_{0},
$$

where $\bar{\mu}:=\mu /(1+\varepsilon)$. We can then rewrite (4a) as

$$
\nabla V(x) \cdot f(x, w) \leq-\bar{c} V(x)-(c-\bar{c}) V(x)+\chi(|w|)
$$

and conclude from Lemma 1 in the Appendix that between any two consecutive impulses $t_{k-1}, t_{k}$ the function $t \mapsto V(x(t))$ is absolutely continuous and

$$
\dot{V}(x(t)) \leq-\bar{c} V(x(t))-(c-\bar{c}) V(x(t))+\chi(|w(t)|),
$$

$\forall t \in\left(t_{k-1}, t_{k}\right)$ a.e. This means that

$$
(c-\bar{c}) V(x(t)) \geq \chi(|w(t)|) \Rightarrow \dot{V}(x(t)) \leq-\bar{c} V(x(t))
$$

$\forall t \in\left(t_{k-1}, t_{k}\right)$ a.e. Similarly, from (4b) we conclude that at every impulse time $t_{k}$

$$
\begin{aligned}
& \left(e^{-\bar{d}}-e^{-d}\right) V\left(x^{-}\left(t_{k}\right)\right) \geq \chi\left(\left|w^{-}\left(t_{k}\right)\right|\right) \\
& \quad \Rightarrow V\left(x\left(t_{k}\right)\right) \leq e^{-\bar{d}} V\left(x^{-}\left(t_{k}\right)\right) .
\end{aligned}
$$

Let $a:=\left(\min \left\{c-\bar{c}, e^{-\bar{d}}-e^{-d}\right\}\right)^{-1}>0$. Because of the right-continuity of $x$ and $w$, there exists a sequence of 
times $t_{0}=: \hat{t}_{0} \leq \check{t}_{1}<\hat{t}_{1}<\check{t}_{2}<\hat{t}_{2}<\ldots$ such that we have

$$
\begin{array}{ll}
V(x(t)) \geq a \chi\left(\|w\|_{\left[t_{0}, t\right]}\right) & \forall t \in\left[\hat{t}_{i}, \check{t}_{i+1}\right), i=0,1, \ldots \\
V(x(t)) \leq a \chi\left(\|w\|_{\left[t_{0}, t\right]}\right) \quad \forall t \in\left[\check{t}_{i}, \hat{t}_{i}\right), \quad i=1,2, \ldots
\end{array}
$$

This sequence of times breaks the interval $\left[t_{0}, \infty\right)$ into a disjoint union of subintervals. Either this sequence is infinite and all subintervals are finite, or the sequence is finite and the last subinterval is infinite. We now analyze these subintervals separately.

Suppose that $\check{t}_{1}>t_{0}$ so that the subinterval $\left[t_{0}, \check{t}_{1}\right)$ is non-empty; otherwise, skip forward to the line below (14). Using (7) and (9a), we conclude that between any two consecutive impulses $t_{k-1}, t_{k} \in\left(t_{0}, \check{t}_{1}\right]$, we have that $\dot{V}(x(t)) \leq-\bar{c} V(x(t)) \forall t \in\left(t_{k-1}, t_{k}\right)$ a.e. Therefore,

$$
V\left(x^{-}\left(t_{k}\right)\right) \leq e^{-\bar{c}\left(t_{k}-t_{k-1}\right)} V\left(x\left(t_{k-1}\right)\right) .
$$

Moreover, in view of (8) and (9a),

$$
V\left(x\left(t_{k}\right)\right) \leq e^{-\bar{d}} V\left(x^{-}\left(t_{k}\right)\right) .
$$

Combining (10) and (11), we conclude that

$$
V\left(x\left(t_{k}\right)\right) \leq e^{-\bar{d}} e^{-\bar{c}\left(t_{k}-t_{k-1}\right)} V\left(x\left(t_{k-1}\right)\right)
$$

Noting that (10) is also true for $k=1$, we can iterate (12) over the $N\left(t, t_{0}\right)$ impulses on $\left(t_{0}, t\right]$ to obtain the bound

$$
V(x(t)) \leq e^{-\bar{d} N\left(t, t_{0}\right)-\bar{c}\left(t-t_{0}\right)} V\left(x\left(t_{0}\right)\right)
$$

$\forall t \in\left(t_{0}, \check{t}_{1}\right]$ (cf. [14, Theorem 1.10.2]). Here we assumed that $\check{t}_{1}<\infty$, otherwise the bound holds on $\left(t_{0}, \infty\right)$. Combining this with (6), we conclude that

$$
V(x(t)) \leq e^{\bar{\mu}-\bar{\lambda}\left(t-t_{0}\right)} V\left(x\left(t_{0}\right)\right) \quad \forall t \in\left(t_{0}, \check{t}_{1}\right] .
$$

Next we show that for $t \geq \check{t}_{1}$, it is possible to construct an upper bound for $\bar{V}(x(t))$ that only depends on $\|w\|_{\left[t_{0}, t\right]}$. On every subinterval of the form $\left[\check{t}_{i}, \hat{t}_{i}\right)$ we already have $(9 \mathrm{~b})$. If $\hat{t}_{i}$ is not an impulse time, then the same bound holds for $t=\hat{t}_{i}$. If $\hat{t}_{i}$ is an impulse time, then (4b) gives

$$
V\left(x\left(\hat{t}_{i}\right)\right) \leq a e^{-d} \chi\left(\|w\|_{\left[t_{0}, \hat{t}_{i}\right)}\right)+\chi\left(\left|w^{-}\left(\hat{t}_{i}\right)\right|\right)
$$

In either case, we have

$$
V(x(t)) \leq\left(a e^{|d|}+1\right) \chi\left(\|w\|_{\left[t_{0}, t\right]}\right) \quad \forall t \in\left[\check{t}_{i}, \hat{t}_{i}\right], i \geq 1,
$$

where again the bound holds $\forall t \geq \check{t}_{i}$ if $\hat{t}_{i}=\infty$. Now consider any subinterval of the form $\left[\hat{t}_{i}, \check{t}_{i+1}\right), i \geq 1$. Repeating the argument used to establish (14), with $\hat{t}_{i}$ in place of $t_{0}$, and using (15) with $t=\hat{t}_{i}$, we obtain

$$
\begin{aligned}
& V(x(t)) \leq e^{\bar{\mu}-\bar{\lambda}\left(t-\hat{t}_{i}\right)} V\left(x\left(\hat{t}_{i}\right)\right) \\
& \leq e^{\bar{\mu}}\left(a e^{|d|}+1\right) \chi\left(\|w\|_{\left[t_{0}, \hat{t}_{i}\right]}\right) \quad \forall t \in\left(\hat{t}_{i}, \check{t}_{i+1}\right], i \geq 1 .
\end{aligned}
$$

Combining this with (14) and (15) and noting that $\bar{\mu}>$ 0 , we finally obtain the following global bound:

$$
\begin{aligned}
V(x(t)) \leq & \max \left\{e^{\bar{\mu}-\bar{\lambda}\left(t-t_{0}\right)} V\left(x\left(t_{0}\right)\right),\right. \\
& \left.e^{\bar{\mu}}\left(a e^{|d|}+1\right) \chi\left(\|w\|_{\left[t_{0}, t\right]}\right)\right\} \quad \forall t \geq t_{0} .
\end{aligned}
$$

The ISS estimate (2) follows from this by standard arguments. Namely, since $V$ is positive definite and radially unbounded, it satisfies $\alpha_{1}(|x|) \leq V(x) \leq \alpha_{2}(|x|)$ for some $\alpha_{1}, \alpha_{2} \in \mathcal{K}_{\infty}$. Therefore, (17) implies (2) with $\beta(r, t):=\alpha_{1}^{-1}\left(e^{\bar{\mu}-\bar{\lambda} t} \alpha_{2}(r)\right)$ and $\gamma(r):=\alpha_{1}^{-1}\left(e^{\bar{\mu}}\left(a e^{|d|}+\right.\right.$ 1) $\chi(r))$. Global existence of solutions also follows from the so-established boundedness of the state. Uniformity is also clear, since the functions $\beta$ and $\gamma$ do not depend on the particular choice of the impulse time sequence.

\section{ISS with (reverse) ADT}

Suppose that an impulsive system has a candidate exponential ISS-Lyapunov function with rate coefficients $c$ and $d$, as in (4). When $d<0$, we must necessarily have $c \geq \lambda>0$ for (5) to hold. In this case, (4a) says that the continuous dynamics $\dot{x}=f(x, w)$ are ISS with respect to $w$. Indeed, the existence of an ISS-Lyapunov function $V$ satisfying $\nabla V(x) \cdot f(x, w) \leq-\alpha(V(x))+\chi(|w|)$ with $\alpha, \chi \in \mathcal{K}_{\infty}$ is equivalent to ISS [24], and taking $\alpha$ to be linear is no loss of generality [21].

Since $d<0$, the impulses can potentially destroy ISS, and we must require that they do not happen too frequently. Not surprisingly, in this case the condition (5) enforces an upper bound on the number of impulses times: for $c=\lambda$ it only holds when the number of impulse times is no larger than $N_{0}:=\mu /|d|$ and for $c>\lambda$ it can be re-written as

$$
N(t, s) \leq \frac{t-s}{\tau^{*}}+N_{0} \quad \forall t \geq s \geq t_{0}
$$

for appropriately defined constants $\tau^{*}, N_{0}>0$. This corresponds to the concept of average dwell-time (ADT) for switched systems introduced in [9]. The special case $N_{0}=1$ reduces to a dwell-time condition in which consecutive impulses must be separated by at least $\tau^{*}$ units of time. 
Conversely, when $c<0$ we must have $d>0$ for (5) to hold. In this case, the condition (4b) says that the discrete dynamics $x(k+1)=g(x(k), w(k))$ are ISS with respect to $w$. Indeed, the existence of an ISS-Lyapunov function $V$ satisfying $V(g(x, w)) \leq-\alpha(V(x))+\chi(|w|)$ with $\alpha, \chi \in \mathcal{K}_{\infty}$ is equivalent to discrete-time ISS [12], and taking $\alpha$ to be linear is no loss of generality ${ }^{5}$. Since $c<0$, the continuous flow can potentially destroy ISS, so we must require flows to be persistently interrupted by impulses. In this case, the condition (5) enforces a lower bound on the number of impulse times and it can be re-written as

$$
N(t, s) \geq \frac{t-s}{\tau^{*}}-N_{0} \quad \forall t \geq s \geq t_{0}
$$

for appropriate $\tau^{*}, N_{0}>0$. This is a reverse $A D T$ condition that demands, on average, at least one impulse per interval of length $\tau^{*}$. We remark that the existence of a function $V$ satisfying (4a) with $c<0$ amounts to forward completeness of the continuous dynamics [1, Corollary 2.11]; of course, we need the same function $V$ to also capture ISS of the discrete dynamics as in (4b).

Let $\mathcal{S}_{\text {avg }}\left[\tau^{*}, N_{0}\right]$ denote the class of ADT impulse time sequences which satisfy (18), and let $\mathcal{S}_{\mathrm{r}-\text { avg }}\left[\tau^{*}, N_{0}\right]$ denote the class of reverse ADT impulse time sequences which satisfy (19). The following result follows from the above observations:

Corollary 1 (ADT ISS) Let $V$ be a candidate exponential ISS-Lyapunov function for (1) with rate coefficients $c, d \in \mathbb{R}$.

(a) When $d<0$ and $c>0$, (1) is uniformly ISS over $\mathcal{S}_{\text {avg }}\left[\tau^{*}, N_{0}\right]$ for all $\tau^{*}>|d| / c$ and $N_{0}>0$.

(b) When $d>0$ and $c<0$, (1) is uniformly ISS over $\mathcal{S}_{\mathrm{r}-\mathrm{avg}}\left[\tau^{*}, N_{0}\right]$ for all $\tau^{*}<d /|c|$ and $N_{0}>0$.

Proof of Corollary 1. To prove (a), pick some $\tau^{*}>|d| / c$, $N_{0}>0$ and take an arbitrary impulse time sequence in $\mathcal{S}_{\text {avg }}\left[\tau^{*}, N_{0}\right]$. In view of (18), we have that $N(t, s) \leq$ $(c-\lambda)(t-s) /|d|+N_{0} \forall t \geq s \geq t_{0}$, for $\lambda:=c-|d| / \tau^{*}>$ 0 , from which we conclude that (5) holds with $\mu:=$ $|d| N_{0}$. Uniform ISS then follows from Theorem 1.

To prove (b), pick some $\tau^{*}<d /|c|$ and take an arbitrary impulse time sequence in $\mathcal{S}_{\mathrm{r}-\text { avg }}\left[\tau^{*}, N_{0}\right]$. In view of (19), we have that $N(t, s) \geq-(c-\lambda)(t-s) / d-N_{0} \forall t \geq s \geq$ $t_{0}$, for $\lambda:=c+d / \tau^{*}>0$, from which we conclude that (5) holds with $\mu:=d N_{0}$. Uniform ISS then follows from Theorem 1.

\footnotetext{
${ }^{5}$ This follows from the implication (b) $\Rightarrow$ (c) in Theorem 2, which will be stated in Section 5 .
}

\section{ISS for arbitrary impulse time sequences}

When the rate coefficients of a candidate exponential ISS-Lyapunov function are both positive, Theorem 1 gives us uniform ISS for arbitrary impulse time sequences, because (5) poses no constraints on the impulse time sequences, as long as we choose $\lambda \leq c$. However, the system actually exhibits a stronger form of uniform ISS.

To state this property we need the following notion: A function $\beta:[0, \infty) \times[0, \infty) \times[0, \infty) \rightarrow[0, \infty)$ is of class $\mathcal{K} \mathcal{L} \mathcal{L}$, and we write $\beta \in \mathcal{K} \mathcal{L} \mathcal{L}$, when $\beta(\cdot, s, \cdot)$ and $\beta(\cdot, \cdot, s)$ are of class $\mathcal{K} \mathcal{L}$ for each fixed $s \geq 0$. We say that the impulsive system (1) is strongly uniformly ISS if there exist functions $\beta \in \mathcal{K} \mathcal{L} \mathcal{L}$ and $\gamma \in \mathcal{K}_{\infty}$ such that for every initial condition, every input $w$, and every sequence of impulse times ${ }^{6}$ the solution to (1) exists globally and satisfies

$$
|x(t)| \leq \beta\left(\left|x\left(t_{0}\right)\right|, N\left(t, t_{0}\right), t-t_{0}\right)+\gamma\left(\|w\|_{\left[t_{0}, t\right]}\right)
$$

$\forall t \geq t_{0}$. We emphasize that this property is stronger than uniform ISS over the set $\mathcal{S}_{\text {all }}$ containing all monotone sequences of impulse times that are finite or unbounded. For example, the one-dimensional impulsive system

$$
\begin{cases}\dot{x}(t)=-x(t)+w(t), & t \neq t_{k}, k \in\{1,2, \ldots\} \\ x(t)=-x^{-}(t), & t=t_{k}, k \in\{1,2, \ldots\}\end{cases}
$$

is uniformly ISS over $\mathcal{S}_{\text {all }}$, but not strongly uniformly ISS. This is because (20) would imply that if we were given a finite time interval $\left[t_{0}, T\right]$, a particular initial condition $x\left(t_{0}\right)$, and the zero input $w(t)=0 \forall t \in\left[t_{0}, T\right]$, then we could make $|x(T)|$ arbitrarily small by increasing the number of impulses $N\left(T, t_{0}\right)$ on $\left(t_{0}, T\right]$. However, with the zero input to $(21)$, the norm of $x(T)$ will be exactly the same regardless of how many impulses take place in $\left(t_{0}, T\right]$.

Remark 1 In the framework of [4], the system (21) would not be ISS because it accepts a solution for which the time sequence $t_{k}$ is infinite but bounded, and such a solution would not converge to zero. It turns out that strong uniform ISS is essentially the same as ISS in the framework of [4], as noted in the proof of Theorem 2 below.

As mentioned above, when the rate coefficients of a candidate exponential ISS-Lyapunov function are both positive, Theorem 1 gives us uniform ISS over $\mathcal{S}_{\text {all }}$, but the system is actually strongly uniformly ISS. Moreover, the existence of such a candidate exponential ISS-Lyapunov function is also a necessary condition for strong uniform

\footnotetext{
${ }^{6}$ Recall that the sequence of impulse times is always strictly increasing and either finite or infinite and unbounded.
} 
ISS. The following result provides a complete characterization of this type of stability.

Theorem 2 (strong uniform ISS) Assume that the impulse map $g$ in (1) is continuous. The following three statements are equivalent:

(a) (1) is strongly uniformly ISS.

(b) There exist a locally Lipschitz, positive definite, radially unbounded function $U: \mathbb{R}^{n} \rightarrow \mathbb{R}$ and $\alpha, \chi \in \mathcal{K}_{\infty}$ that satisfy ${ }^{7}$

$$
\nabla U(x) \cdot f(x, w) \leq-\alpha(U(x))+\chi(|w|) \quad \forall x \text { a.e. }, \forall w
$$

$$
U(g(x, w)) \leq(i d-\alpha)(U(x))+\chi(|w|) \quad \forall x, w .
$$

(c) There exists a candidate exponential ISS-Lyapunov function $V$ for (1) with positive rate coefficients.

From Theorem 2 we conclude that if the impulsive system is strongly uniformly ISS [statement (a)], then both the continuous and discrete dynamics must be ISS [as implied by statement (b)]. However, the converse is not true. In fact, one can even construct impulsive systems for which the continuous and discrete dynamics are both exponentially stable in isolation (without inputs), but the combined impulsive system exhibits unbounded solutions. Such an impulsive system is essentially given in [5, Example 3.1]. This does not contradict Theorem 2 because the statement (b) also asks for the continuous and discrete dynamics to "share" the same ISS-Lyapunov function, which is shown in Theorem 2 to be a necessary condition for strong uniform ISS.

Proof of Theorem 2. (a) $\Rightarrow$ (b) The proof of this implication relies heavily on results from [4], from which we borrow the notation and terminology used to define and characterize properties of hybrid systems. We start by constructing the following hybrid system:

$$
\dot{z}=f(z, u), \quad z \in C, \quad z^{+}=g(z, u), \quad z \in D,
$$

with trivial flow and jump sets $C=D:=\mathbb{R}^{n}$, and with the same functions $f$ and $g$ as in the definition of the impulsive system (1). Such a hybrid system satisfies [4, Standing Assumption 1]. We first show that the hybrid system (23) is forward complete and ISS in the sense of [4], and then use [4, Theorem 2] to conclude that it must have an ISS-Lyapunov function in the sense of [4]. This ISS-Lyapunov function will turn out to satisfy (22a)(22b). The key technical difficulty in this argument arises

\footnotetext{
7 Taking the same functions $\alpha$ and $\chi$ in (22a) and (22b) is no loss of generality, because we could always consider the minimum of the two $\alpha$ 'a and the maximum of the two $\chi$ 's.
}

from the fact that some solutions to the hybrid system (23) do not have direct correspondence to solutions to the impulsive system (1). In particular, one must make sure that ISS of (1) guarantees that even those solutions to the hybrid system (23) for which there are multiple jumps at the same time instant or for which the set of jump times has a finite accumulation point are well behaved. To overcome this difficulty, we use a contradiction argument to show that for a solution to the hybrid system (23) to misbehave, the impulsive system (1) would have to have a (somewhat different, but close) solution that also misbehaves. Due to space limitations, details of this proof are omitted; they can be found in [11].

(b) $\Rightarrow$ (c) To prove this implication we explicitly construct a candidate exponential ISS-Lyapunov function $V$ from a non-exponential one $U$. Let $V(x):=\kappa(U(x))$, where $\kappa \in \mathcal{K}_{\infty}$ is chosen to be continuously differentiable with $\kappa^{\prime}$ nonnegative and nondecreasing, and

$$
\kappa^{\prime}(s) \alpha(s) \geq 2 \kappa(s) \quad \forall s \geq 0 .
$$

Such a function is constructed in [21, pp. 22-23]. From (24) and (22a), we conclude that

$$
\begin{aligned}
& \nabla V(x) \cdot f(x, w)=\kappa^{\prime}(U(x)) \nabla U(x) \cdot f(x, w) \\
& \quad \leq-\kappa^{\prime}(U(x)) \alpha(U(x))+\kappa^{\prime}(U(x)) \chi(|w|) \\
& \quad \leq-V(x)-\kappa^{\prime}(U(x)) \alpha(U(x)) / 2+\kappa^{\prime}(U(x)) \chi(|w|)
\end{aligned}
$$

$\forall x$ a.e., $\forall w$. When $\alpha(U(x)) \leq 2 \chi(|w|)$, we have

$$
\kappa^{\prime}(U(x)) \chi(|w|) \leq \kappa^{\prime} \circ \alpha^{-1}(2 \chi(|w|)) \chi(|w|)=: \bar{\chi}(|w|)
$$

and when $\alpha(U(x))>2 \chi(|w|)$

$$
-\kappa^{\prime}(U(x)) \alpha(U(x)) / 2+\kappa^{\prime}(U(x)) \chi(|w|) \leq 0 .
$$

In either case, we conclude from (25) that

$$
\nabla V(x) \cdot f(x, w) \leq-V(x)+\bar{\chi}(|w|) \quad \forall x \text { a.e., } \forall w
$$

On the other hand, using (24), the Mean Value Theorem, and the fact that $\kappa^{\prime}$ is nondecreasing, we conclude that

$$
\begin{aligned}
2 \kappa(s) \leq \kappa^{\prime}(s) \alpha(s) / 2+ & \kappa(s) \leq \kappa(s+\alpha(s) / 2) \\
& =\kappa \circ(i d+\alpha / 2)(s) \quad \forall s \geq 0 .
\end{aligned}
$$

Specializing this inequality for ${ }^{8} s:=(i d-\alpha / 2)(r), r \geq 0$ and using the fact that $(i d-\alpha / 2)(r) \leq(i d+\alpha / 2)^{-1}(r)$

\footnotetext{
8 The function $i d-\alpha / 2$ must be nonnegative, otherwise we would have a contradiction between $(22 \mathrm{~b})$ and positive definiteness of $U$.
} 
$\forall r \geq 0$, we further obtain

$$
2 \kappa \circ(i d-\alpha / 2)(r) \leq \kappa(r) \quad \forall r \geq 0 .
$$

From the definition of $V$ and (22b), we conclude that

$$
V(g(x, w)) \leq \kappa((i d-\alpha)(U(x))+\chi(|w|))
$$

$\forall x, w$. When $\alpha(U(x)) \leq 2 \chi(|w|)$, we have

$$
\begin{array}{r}
\kappa((i d-\alpha)(U(x))+\chi(|w|) \mid) \leq \kappa(U(x)+\chi(|w|) \mid) \\
\leq \kappa\left(\alpha^{-1}(2 \chi(|w|))+\chi(|w|)\right)=: \tilde{\chi}(|w|)
\end{array}
$$

and when $\alpha(U(x))>2 \chi(|w|)$,

$$
\begin{aligned}
\kappa((i d-\alpha)(U(x)) & +\chi(|w|)) \\
\leq & \kappa \circ(i d-\alpha / 2)(U(x)) \leq V(x) / 2
\end{aligned}
$$

by virtue of (27). In either case, we conclude from (28) that

$$
V(g(x, w)) \leq \frac{1}{2} V(x)+\tilde{\chi}(|w|) \quad \forall x, w .
$$

This finishes the proof since (26) and (29) show that $V$ is a candidate exponential ISS-Lyapunov function $V$ with positive rate coefficients.

(c) $\Rightarrow$ (a) Since $c$ and $d$ are both positive, we can pick some $\lambda>0$ sufficiently small so that (5) holds for every sequence of impulse times. We now proceed as in the proof of Theorem 1 to conclude that

$$
\begin{aligned}
V(x(t)) \leq e^{-\bar{d} N\left(t, t_{0}\right)-\bar{c}\left(t-t_{0}\right)} V\left(x\left(t_{0}\right)\right) & \\
& +e^{\bar{\mu}}\left(a e^{|d|}+1\right) \chi\left(\|w\|_{\left[t_{0}, t\right]}\right) \quad \forall t \geq t_{0} .
\end{aligned}
$$

This expression is obtained in the same way as (17), except that now we combined the bounds (13) and (16) instead of (14) and (16). Since both $\bar{d}$ and $\bar{c}$ are positive, the ISS estimate (20) follows by standard arguments.

Remark 2 (neutral dynamics) It should be clear from the proof of Theorem 2 [implication $(b) \Rightarrow(c)$ ] that if $(22 \mathrm{~b})$ is replaced by $U(g(x, w)) \leq U(x) \forall x, w$, i.e., if the impulses are "neutral" rather than "helpful" for ISS, then there exists a candidate exponential ISSLyapunov function $V$ with rate coefficients $c=1, d=0$ for which the $\chi$ term is absent from (4b) ${ }^{9}$. In this case, it is straightforward to prove that (1) is uniformly ISS

\footnotetext{
9 To check that this is so, note that we can always take $c=1$ as in (26). Moreover, when $U(g(x, w)) \leq U(x) \forall x, w$, the equation (28) holds with $\alpha=\chi=0$, and therefore the right-hand side of $(28)$ is simply $\kappa(U(x))=V(x)$, which corresponds to $d=0$.
}

over $\mathcal{S}_{\text {all }}$, but it may not be strongly uniformly ISS. The example in (21) illustrates this situation.

One can also show that when (22b) holds but the continuous dynamics are "neutral," - i.e., (22a) is replaced by $\nabla V(x) \cdot f(x, w) \leq 0 \forall x$ a.e., $\forall w$ - the impulsive system (1) is uniformly ISS, but only over restricted classes of impulse sequences, for which the number of impulses $N\left(t, t_{0}\right)$ in the interval $\left(t_{0}, t\right]$ is bounded from below by an expression of the form $N\left(t, t_{0}\right) \geq \eta\left(t-t_{0}\right) \forall t>t_{0}$, for some function $\eta \in \mathcal{K}_{\infty}$.

More interesting situations arise when "neutrality" only holds for the zero input. This case is treated more thoroughly in the next section.

\section{ISS for arbitrary ADT}

We have just seen in Section 5 that when the continuous and the discrete dynamics are both ISS and share an ISSLyapunov function, we have a strong notion of uniform ISS for the impulsive system. When only one of these dynamics is ISS and the other is unstable, the results in Section 4 tell us that some form of ADT is needed for ISS. We now consider the "marginal" case in which one of the dynamics is ISS and the other one is only marginally stable for the zero input.

Consider the impulsive system (1) and assume that $\dot{x}=f(x, w)$ is continuous-time ISS, but $x(k+1)=$ $g(x(k), w(k))$ is only marginally stable for the zero input $w(k)=0 \forall k$. This type of systems occurs, e.g., in $[16$, Section 4$]$ in the context of control with limited information or in the MEMS device that we discuss in Section 8.1. For such systems, one should not expect uniform ISS over $\mathcal{S}_{\text {all }}$ because the discrete dynamics lack ISS, but (4b) will typically still hold for some $d<0$ and part (a) of Corollary 1 thus provides an ISS result in terms of a minimum ADT $\tau^{*}>|d| / c$. However, for these systems one can often draw stronger conclusions because $d$ can be made arbitrarily close to zero. Motivated by this observation, we say that the impulsive system (1) is ISS for arbitrarily small ADT when it is uniformly ISS over every class $\mathcal{S}_{\text {avg }}\left[\tau^{*}, N_{0}\right]$ of ADT impulse time sequences that satisfy (18) with $\tau^{*}>0, N_{0}<\infty$.

Alternatively, we consider an impulsive system for which $x(k+1)=g(x(k), w(k))$ is discrete-time ISS, but $\dot{x}=f(x, w)$ is only marginally stable for the zero input $w(t)=0 \forall t$. This type of systems occurs, e.g., in the context of networked control systems such as the one described in Section 8.2 or in $[28]^{10}$. For such systems

\footnotetext{
${ }^{10}$ The analysis in [28] deals with stochastic disturbances $w$ and considers more general vector fields. A deterministic version of the framework in [28] with marginally stable processes leads to the class of systems considered here.
} 
(4a) will typically hold for some $c<0$ and part (b) of Corollary 1 provides an ISS result in terms of a maximum reverse a.d.t $\tau^{*}:=d /(-c), \lambda>0$. However, also here one can draw stronger conclusions because typically $c$ can be made arbitrarily close to zero. Motivated by this, we say that the impulsive system (1) is ISS for arbitrarily large reverse $A D T$ when it is uniformly ISS over every class $\mathcal{S}_{\mathrm{r}-\mathrm{avg}}\left[\tau^{*}, N_{0}\right]$ of reverse ADT impulse time sequences that satisfy (19) with $\tau^{*}>0, N_{0}<\infty$.

It turns out that for both cases discussed above, we need a little more than marginal stability for the sub-system that is not ISS, and therefore we introduce the following terminology: We say that $V: \mathbb{R}^{n} \rightarrow \mathbb{R}$ is non-expansive for the impulse map $g$ when $V$ is positive definite, radially unbounded, and for every $d<0$ there exists a function $\chi \in \mathcal{K}_{\infty}$ for which (4b) holds. This terminology is motivated by the observation that such a function must necessarily satisfy $V(g(x, 0)) \leq V(x) \forall x$. Its existence thus guarantees marginal stability of the discrete dynamics $x(k+1)=g(x(k), 0)$ with zero input, but it actually provides more than that. However, it is not sufficient for ISS of $x(k+1)=g(x(k), w(k))$ with respect to a nonzero input $w(k)$.

Alternatively, we say that a locally Lipschitz function $V$ is non-expansive for the vector field $f$ when $V$ is positive definite, radially unbounded, and for every $c<0$ there exists a function $\chi \in \mathcal{K}_{\infty}$ for which (4a) holds. Such a function also satisfies $\nabla V(x) \cdot f(x, 0) \leq 0 \forall x$ a.e. and it therefore guarantees marginal stability of the continuous dynamics $\dot{x}=f(x, 0)$ for the zero input, but it is not enough to guarantee ISS of $\dot{x}=f(x, w)$ with respect to $w$.

The following result follows from Corollary 1:

Corollary 2 (ISS for arbitrary ADT) Let $V$ be a candidate exponential ISS-Lyapunov function for (1) with rate coefficients $c, d \in \mathbb{R}$.

(a) When $c>0$ and $V$ is non-expansive for the impulse mapg, (1) is uniformly ISS for arbitrarily small $A D T$.

(b) When $d>0$ and $V$ is non-expansive for the vector field $f,(1)$ is uniformly ISS for arbitrarily large reverse $A D T$.

The remainder of this section is devoted to the question of whether or not a given function $V$ is non-expansive.

\subsection{Non-expansiveness for impulse maps}

To state the following result, we say that a function $h$ : $\mathbb{R}^{n} \times \mathbb{R}^{m} \rightarrow \mathbb{R}^{n}$ has class- $\mathcal{K}$ growth in $w$ uniformly over $x$ if

$$
|h(x, w)| \leq \gamma(|w|) \quad \forall x, w
$$

for some function $\gamma \in \mathcal{K}$, which we call the growth estimate for $h$. The following result (proved in the appendix) provides simple conditions that can be used to establish that a candidate Lyapunov function is non-expansive for a given impulse map.

Theorem 3 (non-expansiveness for impulse maps) Consider a locally Lipschitz, positive definite, radially unbounded function $V: \mathbb{R}^{n} \rightarrow \mathbb{R}$ and an impulse map $g$ for which $V(g(x, 0)) \leq V(x) \forall x$ and $g(x, w)-g(x, 0)$ has class- $\mathcal{K}$ growth in $w$ uniformly over $x$. The function $V$ is non-expansive for the impulse map $g$ if either of the following conditions holds:

C1 $V$ is a positive-coefficient linear combination of functions that are non-expansive for the impulse map g.

C2 $V(x) /|\nabla V(x)|$ is radially unbounded a.e.

C3 $V$ is a positive-coefficient linear combination of homogeneous functions ${ }^{11}$ with (possibly different) degrees larger than or equal to one.

Remark 3 Theorem 3 describes a very broad class of positive definite functions $V$ that are non-expansive. Indeed, C2 simply requires that the gradient of $V$ be dominated by $V$ itself and $C 3$ says that, e.g., any polynomial function falls in this class. One may then wonder if in practice one will ever encounter ISS impulsive systems with candidate exponential ISS-Lyapunov functions that do not exhibit the non-expansiveness property. The answer is affirmative and a simple one-dimensional example is given by

$$
\begin{cases}\dot{x}=-\operatorname{sat}(x), & t \neq t_{k}, k=1,2, \ldots \\ x=x^{-}+\operatorname{sat}\left(w^{-}\right), & t=t_{k}, k=1,2, \ldots,\end{cases}
$$

where sat $(\cdot)$ denotes the saturation function limited at \pm 1 and with unit slope on $[-1,1]$. This system is uniformly ISS over the class of ADT impulse time sequences that satisfy (18) for any $\tau^{*}>1$ because

$$
V(x):= \begin{cases}x^{2} & |x| \leq 1 \\ e^{2(|x|-1)} & |x|>1\end{cases}
$$

is a candidate exponential ISS-Lyapunov function with rate coefficients $c=2$ and $d=-2$. However, it is not ISS for arbitrarily small ADT since $x$ can explode with bounded inputs, provided that the impulse times are closely spaced. As expected, the function (30) does not satisfy any of the conditions in Theorem 3.

The next Corollary covers a useful class of systems, which includes the case of asymptotically stable linear continuous dynamics and marginally stable linear discrete dynamics.

\footnotetext{
${ }^{11}$ We recall that a function $V: \mathbb{R}^{n} \rightarrow \mathbb{R}$ is homogeneous of degree $p$ if $V(\lambda x)=\lambda^{p} V(x) \forall \lambda \geq 0, x \in \mathbb{R}^{n}$.
} 
Corollary 3 (GES vs. ISS for arbitrarily small ADT) Impulsive systems of the following form are always ISS for arbitrarily small $A D T$ :

$$
\begin{cases}\dot{x}=f_{1}(x)+f_{2}(w), & t \neq t_{k}, k=1,2, \ldots \\ x=x^{-}+g\left(x^{-}, w\right), & t=t_{k}, k=1,2, \ldots\end{cases}
$$

where $\dot{x}=f_{1}(x)$ is globally exponentially stable, $f_{1}$ is globally Lipschitz, $f_{2}(0)=0$, and $g(x, w)$ has class $-\mathcal{K}$ growth in $w$ uniformly over $x$.

Proof of Corollary 3. [13, Theorem 4.14] guarantees the existence of a locally Lipschitz function $V$ satisfying $a_{1}|x|^{2} \leq V(x) \leq a_{2}|x|^{2}, \nabla V(x) \cdot f_{1}(x) \leq-a_{3}|x|^{2}$, and $|\nabla V(x)| \leq a_{4}|x|$ a.e., where $a_{i}>0, i=1,2,3,4$. This implies that

$$
\nabla V(x) \cdot\left(f_{1}(x)+f_{2}(w)\right) \leq-a_{3}|x|^{2}+a_{4}|x|\left|f_{2}(w)\right|,
$$

from which (4a) follows by square completion, for an appropriately chosen rate coefficient $c>0$. On the other hand, since $g(x, w)$ has class- $\mathcal{K}$ growth in $w$ uniformly over $x$, we must have $g(x, 0)=0 \forall x$, from which we conclude that $V(x+g(x, 0))=V(x)$ and that $(x+g(x, w))-$ $(x+g(x, 0))=g(x, w)$ has class- $\mathcal{K}$ growth. This allows us to use condition $\mathrm{C} 2$ in Theorem 3 to conclude that $V$ is non-expansive for the impulse map $x+g(x, w)$ and the result then follows from Corollary 2.

\subsection{Non-expansiveness for vector fields}

The following result (proved in the appendix) provides simple conditions for establishing that a candidate Lyapunov function is non-expansive for a given vector field.

Theorem 4 (non-expansiveness for vector fields) Consider a locally Lipschitz, positive definite, radially unbounded function $V: \mathbb{R}^{n} \rightarrow \mathbb{R}$ and a vector field $f$ for which $\nabla V(x) \cdot f(x, 0) \leq 0 \forall x$ and $f(x, w)-f(x, 0)$ has class- $\mathcal{K}$ growth in $w$ uniformly over $x$. The function $V$ is non-expansive for the vector field $f$ if either of the following conditions holds:

C4 $V$ is a positive-coefficient linear combination of functions that are non-expansive for the vector field $f$.

C5 $V(x) /|\nabla V(x)|$ is radially unbounded a.e.

C6 $V$ is a positive-coefficient linear combination of homogeneous functions with (possibly different) degrees larger than or equal to one.

The next Corollary covers a useful class of systems, which includes the case of asymptotically stable linear discrete dynamics and marginally stable linear continuous dynamics.
Corollary 4 (GES vs. ISS for arbitrary large rev. ADT) Impulsive systems of the following form are always ISS for arbitrarily large reverse ADT:

$$
\begin{cases}\dot{x}=f(x, w), & t \neq t_{k}, k=1,2, \ldots \\ x=g_{1}\left(x^{-}\right)+g_{2}\left(w^{-}\right), & t=t_{k}, k=1,2, \ldots\end{cases}
$$

where $x(k+1)=g_{1}(x(k))$ is globally exponentially stable, $g_{1}$ is globally Lipschitz, $g_{2}(0)=0$, and $f(x, w)$ has class$\mathcal{K}$ growth in $w$ uniformly over $x$.

Proof of Corollary 4. Under the assumptions of the theorem, it is not difficult to verify that, for some sufficiently large integer $k>0$, there exists $a_{1} \geq 1$ such that the function $V(x):=\sum_{i=0}^{k}|\phi(i, x)|$ is globally Lipschitz and satisfies

$$
\begin{aligned}
& |x| \leq V(x) \leq a_{1}|x|, \\
& V\left(g_{1}(x)\right)-V(x) \leq-|x| / 2,
\end{aligned}
$$

where $\phi\left(k, x_{0}\right)$ denotes the solution to $x(k+1)=$ $g_{1}(x(k))$ at time $k$ starting at $x(0)=x_{0}$. Since $f(x, w)$ has class- $\mathcal{K}$ growth in $w$ uniformly over $x$, we have that $f(x, 0)=0$ and therefore $\nabla V(x) \cdot f(x, 0)=0$ and $f(x, w)-f(x, 0)$ has class- $\mathcal{K}$ growth in $w$ uniformly over $x$. We can then use C5 in Theorem 4 to conclude that $V$ is non-expansive for the vector field $f$. Condition C5 holds because of (31) and the fact that, since $V$ is globally Lipschitz, there exists a constant $a_{2}>0$ for which $|\nabla V(x)| \leq a_{2}$ a.e. We now use Theorem 3 to show that $V$ satisfies (4b) for some rate coefficient $d>0$. To this end, we consider the auxiliary impulsive map $\bar{g}(\bar{x}, w):=\bar{x}+g_{2}(w) \forall \bar{x} \in \mathbb{R}^{n}, w \in \mathbb{R}^{m}$. Since $g_{2}(0)=0$, we have that $V(\bar{g}(\bar{x}, 0))=V(\bar{x})$ $\forall \bar{x}$ and $\bar{g}(\bar{x}, w)-\bar{g}(\bar{x}, 0)=g_{2}(w)$ has class- $\mathcal{K}$ growth in $w$ uniformly over $x$. Since we already established that condition $\mathrm{C} 2$ holds, we conclude that $V$ is nonexpansive for the impulse map $\bar{g}$ and therefore, for every $\bar{d}<0$, we can find an appropriate $\bar{\chi} \in \mathcal{K}_{\infty}$, so that $V(\bar{g}(\bar{x}, w)) \leq e^{-d} V(\bar{x})+\bar{\chi}(|w|) \forall \bar{x}, w$. In particular, for $\bar{x}=g_{1}(x)$ and using (31)-(32), we obtain

$$
\begin{gathered}
V\left(g_{1}(x)+g_{2}(w)\right) \leq e^{-\bar{d}} V\left(g_{1}(x)\right)+\bar{\chi}(|w|) \leq e^{-\bar{d}}(V(x) \\
-|x| / 2)+\bar{\chi}(|w|) \leq e^{-\bar{d}}\left(1-1 /\left(2 a_{1}\right)\right) V(x)+\bar{\chi}(|w|),
\end{gathered}
$$

from which (4b) follows for some $d>0$, provided that we choose $\bar{d}<0$ sufficiently close to zero so that $e^{-\bar{d}}(1-$ $\left.1 /\left(2 a_{1}\right)\right)<1$. The result then follows from Corollary 2 .

\section{Sufficient conditions for integral-ISS}

We now provide iISS counterparts to Theorem 1 and the sufficient condition in Theorem 2. The first result establishes iISS for suitably constrained impulse time 
sequences under the hypotheses of Theorem 1, and the second one establishes iISS for arbitrary impulse time sequences under hypotheses weaker than (22a)-(22b).

Theorem 5 (uniform iISS) Let all hypotheses of Theorem 1 hold and define the class of impulse time sequences $\mathcal{S}[\mu, \lambda], \mu, \lambda>0$ also as in Theorem 1. Then the system (1) is uniformly iISS over $\mathcal{S}[\mu, \lambda]$.

Proof of Theorem 5. From (4a) and (4b) we see that $V(x(t))$ is bounded from above by the (nonnegative) solution $v(t)$ of the impulsive system

$$
\begin{cases}\dot{v}=-c v+\chi(|w|), & t \neq t_{k}, k=1,2, \ldots \\ v=e^{-d} v^{-}+\chi\left(\left|w^{-}\right|\right), & t=t_{k}, k=1,2, \ldots\end{cases}
$$

with the initial condition $v\left(t_{0}\right)=V\left(x\left(t_{0}\right)\right)$. Let $z(t)$ be the (nonnegative and non-decreasing) solution to

$$
\begin{cases}\dot{z}=\chi(|w|), & t \neq t_{k}, k=1,2, \ldots \\ z=z^{-}+\chi\left(\left|w^{-}\right|\right), & t=t_{k}, k=1,2, \ldots\end{cases}
$$

with the initial condition $z\left(t_{0}\right)=0$. Define $y(t):=v(t)-$ $z(t)$. Then $y$ satisfies $y\left(t_{0}\right)=V\left(x\left(t_{0}\right)\right)$ and

$$
\begin{cases}\dot{y}=-c v=-c y-c z, & t \neq t_{k} \\ y=e^{-d} v^{-}-z^{-}=e^{-d} y^{-}-\left(1-e^{-d}\right) z^{-}, & t=t_{k}\end{cases}
$$

Arguing as in the proof of Theorem 1, with $y$ and $z$ playing the roles of $V$ and $w$, respectively, we can show that this impulsive system is ISS with respect to $z$ with linear gain:

$$
y(t) \leq \beta\left(y\left(t_{0}\right), t-t_{0}\right)+\gamma z(t)
$$

for some function $\beta \in \mathcal{K} \mathcal{L}$ and constant $\gamma>0$. Collecting the above facts, we obtain

$$
\begin{gathered}
V(x(t)) \leq v(t)=y(t)+z(t) \leq \beta\left(y\left(t_{0}\right), t-t_{0}\right)+(\gamma+1) z \\
=\beta\left(V\left(x\left(t_{0}\right)\right), t-t_{0}\right)+\int_{t_{0}}^{t}(\gamma+1) \chi(|w(s)|) d s \\
+\sum_{t_{k} \in\left[t_{0}, t\right]}(\gamma+1) \chi\left(\left|w^{-}\left(t_{k}\right)\right|\right)
\end{gathered}
$$

from which the iISS estimate (3) follows.

Theorem 6 (uniform iISS for arbitrary sequences) Suppose that there exists a positive definite, radially unbounded, locally Lipschitz function $V: \mathbb{R}^{n} \rightarrow \mathbb{R}$, a positive definite function $\alpha$, and a class $\mathcal{K}_{\infty}$ function $\chi$ satisfying

$$
\begin{gathered}
\nabla V(x) \cdot f(x, w) \leq-\alpha(V(x))+\chi(|w|) \quad \forall x \text { a.e., } \forall w \\
V(g(x, w)) \leq V(x)+\chi(|w|) \quad \forall x, w .
\end{gathered}
$$

Then the system (1) is uniformly iISS over $\mathcal{S}_{\text {all }}$.

Note that this theorem does not require $\alpha$ to be radially unbounded. This and the relaxation of (22b) to (34b) are the key differences with respect to the necessary and sufficient conditions for strong uniform ISS in Theorem 2.

Proof of Theorem 6. This is a relatively straightforward extension of the proof of the corresponding result for continuous systems given in [2]. From (34a) and (34b) we see that $V(x(t))$ is bounded from above by the (nonnegative) solution $v(t)$ of the impulsive system

$$
\begin{cases}\dot{v}=-\alpha(v)+\chi(|w|), & t \neq t_{k}, k=1,2, \ldots \\ v(t)=v^{-}(t)+\chi\left(\left|w^{-}(t)\right|\right), & t=t_{k}, k=1,2, \ldots\end{cases}
$$

with the initial condition $v\left(t_{0}\right)=V\left(x\left(t_{0}\right)\right)$. Define $z$ and $y$ exactly as in the proof of Theorem 5. Then $y$ is continuous everywhere, non-increasing, and we have

$$
\dot{y}=-\alpha(v)=-\alpha(y+z), \quad y\left(t_{0}\right)=V\left(x\left(t_{0}\right)\right) .
$$

Repeating the argument used to prove [2, Lemma IV.2] we then conclude ${ }^{12}$ that $y$ satisfies (33) with $\beta \in \mathcal{K} \mathcal{L}$ and $\gamma=1$, and iISS follows as before.

\section{Examples}

\subsection{MEMS oscillator}

The displacement $y$ of Micro-Electro-Mechanical System (MEMS) oscillators can be modeled by the following two-dimensional system:

$$
m \ddot{y}+b \dot{y}+k_{1} y+k_{3} y^{3}=v,
$$

where $b$ is a damping coefficient, $k_{1}$ and $k_{3}$ linear and cubic stiffness terms for the restitution force, and $v$ a driving force. Figure 1 shows a schematic of such a device and typical parameters values.

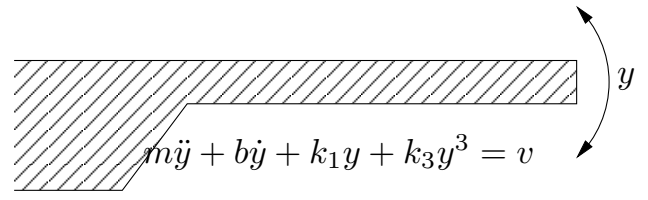

Fig. 1. MEMS device in Example 8.1. The following parameter values are consistent with the folded spring device in [30]: $m=277, b=0.678, k_{1}=7.61$, and $k_{3}=.0441$ (in units of $\mu$ Newtons, $\mu$ meters, $\mu$ seconds, and Volts).

\footnotetext{
${ }^{12}$ That lemma is stated for continuous $z$, but continuity of $z$ is not actually needed in the proof in [2].
} 
For experiments in air, Brownian motion due to collisions with air molecules is the main noise source in masssensing applications $[18,29]$. The transfer of kinetic energy between an air molecule and the oscillator due to a collision at time $t_{k}$ can be modeled by a state-impulse of the following form:

$$
y\left(t_{k}\right)=y^{-}\left(t_{k}\right), \quad \dot{y}\left(t_{k}\right)=\dot{y}^{-}\left(t_{k}\right)+n^{-}\left(t_{k}\right),
$$

where $n^{-}\left(t_{k}\right)$ denotes the change in velocity due to a collision at time $t_{k}$. Defining

$$
V(y, \dot{y}):=y^{2}+0.1 y \dot{y}+36.5 \dot{y}^{2}+18.25 k_{3} y^{4} / m,
$$

we have that (4a) holds with $c=10^{-3}$ and $\chi(|v|):=$ $v^{2} / 2$. Moreover, in view of condition C3 in Theorem 3, the function (37) is non-expansive for the impulse map (36). We thus conclude from Corollary 2 that the impulsive system (35)-(36) is ISS for arbitrarily small ADT. This means that the system remains ISS no matter how frequently the collisions occur. The candidate Lyapunov function (37) was found numerically, using the SOSTOOLS MATLAB toolbox [20].

\subsection{Networked control system}

Consider $n$ one-dimensional linear systems of the form

$$
\dot{x}_{i}=a_{i} x_{i}+b_{i} \nu_{i}, \quad y_{i}=x_{i}+\mu_{i}, \quad i \in\{1,2, \ldots, n\},
$$

where the $\nu_{i}$ denote input disturbances and the $\mu_{i}$ denote measurement/quantization noise. To build a remote estimate of all the $x_{i}$, one is allowed to send one measurement at each time instant $\left\{t_{1}, t_{2}, \ldots\right\}$. Between the reception of measurements the estimate $\hat{x}_{i}$ of $x_{i}$ evolves according to $\dot{\hat{x}}_{i}=a_{i} \hat{x}_{i}, t \notin\left\{t_{1}, t_{2}, \ldots\right\}$ and, denoting by $i_{k}$ the index of the measurement $y_{i_{k}}$ that is sent at time $t_{k}$, we have

$$
\hat{x}_{i}\left(t_{k}\right)= \begin{cases}y_{i_{k}}^{-}\left(t_{k}\right) & i=i_{k} \\ \hat{x}_{i}^{-}\left(t_{k}\right) & i \neq i_{k} .\end{cases}
$$

The dynamics of the resulting estimation error $e_{i}:=\hat{x}_{i}-$ $x_{i}$ can be described by the following impulsive system:

$$
\begin{aligned}
& \dot{e}_{i}=a_{i} e-b_{i} \nu_{i}, \quad t \neq t_{k}, k=1,2, \ldots \\
& e_{i}=\left\{\begin{array}{ll}
\mu_{i_{k}}^{-}\left(t_{k}\right) & i=i_{k} \\
e_{i}^{-}\left(t_{k}\right) & i \neq i_{k},
\end{array} \quad t=t_{k}, k=1,2, \ldots\right.
\end{aligned}
$$

We consider a TOD-like protocol $[19,27]$ to decide which measurement to send: $i_{k}$ is the index corresponding to the largest absolute value of the error $\hat{x}_{i}^{-}-y_{i}^{-}=e_{i}^{-}-\mu_{i}^{-}$, i.e.,

$$
\left|e_{i_{k}}^{-}-\mu_{i_{k}}^{-}\right| \geq\left|e_{i}^{-}-\mu_{i}^{-}\right| \quad \forall i \in\{1,2, \ldots, n\} .
$$

Defining

$$
V(e):=\sum_{i=1}^{n}\left|e_{i}\right|^{2}
$$

it can be shown (see [11]) that for every constant $d \in$ $(0, \log (n / n-1))$ one can find a function $\chi \in \mathcal{K}_{\infty}$ such that

$$
V\left(e\left(t_{k}\right)\right) \leq e^{-d} V\left(e^{-}\left(t_{k}\right)\right)+\chi\left(\left|\mu^{-}\left(t_{k}\right)\right|\right),
$$

where $e$ and $\mu$ are $n$-vectors obtained by stacking together all the $e_{i}$ and $\mu_{i}$, respectively.

We consider three possible cases: (i) When some of the systems (38) are unstable - i.e., some $a_{i}>0$ - the function $V$ in (41) is a candidate exponential ISS-Lyapunov function with rate coefficients

$$
0<d<\log \frac{n}{n-1}, \quad c<-\max _{i} a_{i}<0 .
$$

We then conclude from Corollary 1 that the error system (39) is uniformly ISS over the class of reverse ADT impulse time sequences $\mathcal{S}_{\mathrm{r}-\operatorname{avg}}\left[\tau^{*}, N_{0}\right]$, for all $N_{0}>0$ and

$$
\tau^{*}<\frac{1}{\max _{i} a_{i}} \log \frac{n}{n-1}
$$

In the context of this example this means that measurements must be transmitted at a minimum "average" rate consistent with (43). (ii) When all the systems (38) are stable, but not necessarily asymptotically - i.e., all $a_{i} \leq 0$ - we conclude from Condition C6 in Theorem 4 that the function $V$ is non-expansive for the vector field (39a). In this case, the error system (39) is ISS for arbitrarily large reverse ADT because of Corollary 2. For this example this means that measurements can be transmitted at any positive "average" rate. (iii) When all the systems (38) are asymptotically stable - i.e., all $a_{i}<0$ - the function $V$ in (41) is a candidate exponential ISSLyapunov function with positive rate coefficients. We now conclude from Theorem 2 that the error system (39) is strongly uniformly ISS. This means that there are no constraints posed on the frequency of measurements.

\section{Conclusions}

We introduced the concepts of ISS and integral-ISS for impulsive systems and provided Lyapunov-based sufficient conditions for establishing these properties.

When both the continuous and discrete dynamics are stabilizing, the Lyapunov-based conditions proved to be necessary and sufficient for strong uniform ISS, but it is unclear whether the conditions derived are also tight for the other cases. Investigating this issue is a topic 
for future research. Another topic is the development of numerically efficient procedures for constructing ISSLyapunov functions for impulsive systems, perhaps relying on semi-definite programming.

\section{A Appendix}

Lemma 1 Consider two continuous functions $f: \mathbb{R}^{n} \times$ $\mathbb{R}^{m} \rightarrow \mathbb{R}^{n}, \alpha: \mathbb{R}^{n} \times \mathbb{R}^{m} \rightarrow \mathbb{R}$ and a locally Lipschitz function $V: \mathbb{R}^{n} \rightarrow \mathbb{R}$, with the property that

$$
\nabla V(x) \cdot f(x, w) \leq \alpha(x, w) \quad \forall x \in \mathbb{R}^{n} \backslash \Omega, w \in \mathbb{R}^{m}
$$

for some zero Lebesgue-measure set $\Omega \subset \mathbb{R}^{n}$ that contains all points at which $x \mapsto V(x)$ is not differentiable. For every absolutely continuous solution $x:\left[t_{0}, t_{1}\right) \rightarrow \mathbb{R}^{n}$ to $\dot{x}=f(x, w)$, with $w(t) \in \mathbb{R}^{m}$ locally bounded and Lebesgue-measurable; we have that $t \mapsto V(x(t))$ is absolutely continuous and

$$
\dot{V}(x(t)) \leq \alpha(x(t), w(t)), \quad \forall t \in\left[t_{0}, t_{1}\right) \backslash \mathcal{T}
$$

for some zero Lebesgue-measure set $\mathcal{T} \subset\left[t_{0}, t_{1}\right)$ that contains all points at which $t \mapsto V(x(t))$ is not differentiable.

This result is proved in [26, Section 2]. We emphasize that (A.2) holds even when $x(t), t \in\left[t_{0}, t_{1}\right)$ lies entirely in a subset of $\Omega$ for which (A.1) does not hold because the gradient $\nabla V(x)$ does not exist.

Proof of Theorem 3. C1. We prove this result for a linear combination $V=\alpha_{1} V_{1}+\alpha_{2} V_{2}, \alpha_{1}, \alpha_{2}>0$ of two functions $V_{1}, V_{2}: \mathbb{R}^{n} \rightarrow \mathbb{R}$ both non-expansive for the impulse map $g$ and the general result follows by induction on the number of functions. Since both $V_{1}$ and $V_{2}$ are non-expansive, for any given $d<0$ there exist functions $\chi_{1}, \chi_{2} \in \mathcal{K}_{\infty}$ for which

$$
V_{i}(g(x, w)) \leq e^{-d} V_{i}(x)+\chi_{i}(|w|) \quad \forall x, w, i \in\{1,2\}
$$

and therefore $V$ satisfies (4b) with $\chi:=\alpha_{1} \chi_{1}+\alpha_{2} \chi_{2} \in$ $\mathcal{K}_{\infty}$

C2. For an arbitrary $d<0$, consider the auxiliary function

$$
\kappa_{d}(s):=\min \left\{\frac{s}{2},|d| \underset{|z| \geq s / 2, \nabla V(z) \neq 0}{\operatorname{ess} \inf _{|\nabla V(z)|}} \frac{V(z)}{\mid \nabla V .}\right.
$$

By construction $\kappa_{d}$ is positive for $s \neq 0$, monotone nondecreasing, and radially unbounded. The latter property is due to the hypothesis that $V(z) /|\nabla V(z)|$ is radially unbounded. The above construction does not guarantee that $\kappa_{d} \in \mathcal{K}_{\infty}$, because it may not be continuous or strictly increasing. However, for simplicity we assume that $\kappa_{d} \in \mathcal{K}_{\infty}$, because if this is not the case, we can always replace it by a smaller function in $\mathcal{K}_{\infty}$.

Pick arbitrary $x \in \mathbb{R}^{n}, w \in \mathbb{R}^{m}$ for which $v:=g(x, w)-$ $g(x, 0)$ is "small" in the sense that

$$
|v|=|g(x, w)-g(x, 0)|<\kappa_{d}(|g(x, 0)|)
$$

and define $z(\tau):=g(x, 0)+\tau v \forall \tau \in[0,1]$. Because of (A.4) and the fact that $\kappa_{d}(s) \leq s / 2 \forall s \geq 0$, we have that

$$
\begin{aligned}
|z(\tau)| & \geq|g(x, 0)|-|v| \\
& \geq|g(x, 0)|-\kappa_{d}(|g(x, 0)|) \geq|g(x, 0)| / 2
\end{aligned}
$$

$\forall \tau \in[0,1]$. On the other hand, since $V$ is locally Lipschitz, from Rademacher's Theorem we conclude that $V$ is differentiable almost everywhere and again using (A.4), we obtain $\nabla V(\bar{z}) \cdot v \leq|\nabla V(\bar{z})| \kappa_{d}(|g(x, 0)|) \forall \bar{z}$ a.e. If we further restrict our attention to points for which $|\bar{z}| \geq \frac{|g(x, 0)|}{2}$, from the definition of $\kappa_{d}$ we conclude that

$$
|\bar{z}| \geq|g(x, 0)| / 2 \Rightarrow \nabla V(\bar{z}) \cdot v \leq|d| V(\bar{z}) \quad \forall \bar{z} \text { a.e. }
$$

Since $\frac{\mathrm{d} z}{\mathrm{~d} \tau}=v \forall \tau \in[0,1]$ and $z(\tau)$ satisfies (A.5), we can use (A.6) and Lemma 1 to conclude that $\frac{\partial V(z(\tau))}{\partial \tau} \leq$ $|d| V(z(\tau)) \forall \tau$ a.e., which leads to

$$
\begin{array}{r}
V(g(x, w))=V(z(1)) \leq e^{|d|} V(z(0)) \\
=e^{-d} V(g(x, 0)) \leq e^{-d} V(x),
\end{array}
$$

where we used the fact that $V(g(x, 0)) \leq V(x)$.

Suppose now that we pick $x \in \mathbb{R}^{n}, w \in \mathbb{R}^{m}$ for which $v:=g(x, w)-g(x, 0)$ is "large" in the sense that it satisfies

$$
|g(x, 0)| \leq \kappa_{d}^{-1}(|v|)=\kappa_{d}^{-1}(|g(x, w)-g(x, 0)|)
$$

instead of (A.4). We now have

$$
\begin{aligned}
V(g(x, w))= & V(v+g(x, 0)) \leq \alpha(|v|+|g(x, 0)|) \\
& \leq \alpha \circ\left(i d+\kappa_{d}^{-1}\right)(|v|) \leq \chi(|w|)
\end{aligned}
$$

where $\alpha$ is a class- $\mathcal{K}_{\infty}$ function with the property that $V(x) \leq \alpha(|x|) \forall x \in \mathbb{R}^{n}, \chi:=\alpha \circ\left(i d+\kappa_{d}^{-1}\right) \circ \gamma$, and $\gamma$ is the growth estimate of $g(x, w)-g(x, 0)$. The existence of $\alpha \in \mathcal{K}_{\infty}$ is guaranteed by the fact that $V$ is positive definite and radially unbounded. Combining (A.7) with (A.8), we conclude that (4b) holds for every $x$ and $w$, from which $\mathrm{C} 2$ follows.

C3. In view of $\mathrm{C} 1$, it suffices to show that a homogeneous function $V$ of degree $p \geq 1$ is non-expansive. Suppose 
that we pick an arbitrary $x \in \mathbb{R}^{n}$ for which $\nabla V(x)$ exists and is nonzero and define $\bar{x}:=x /|x|$. It is well known that if $V$ is homogeneous of degree $p \geq 1$, then its gradient $\nabla V$ is homogeneous of degree $p-1 \geq 0$, from which we conclude that

$$
\frac{V(x)}{|\nabla V(x)|}=\frac{|x|^{p} V(\bar{x})}{|x|^{p-1}|\nabla V(\bar{x})|}=\frac{|x| V(\bar{x})}{|\nabla V(\bar{x})|}
$$

so $V(x) /|\nabla V(x)| \rightarrow \infty$ as $|x| \rightarrow \infty$, because $|\nabla V(\bar{x})|$ for $|\bar{x}|=1$ must remain bounded. We thus conclude that $V$ satisfies $\mathrm{C} 2$, which finishes the proof.

Proof of Theorem 4. C4. Similar to the proof of C1 above.

C5. For an arbitrary $c<0$, consider the auxiliary function

$$
\kappa_{c}(s):=-c \operatorname{essinf}_{|z| \geq s, \nabla V(z) \neq 0} \frac{V(z)}{|\nabla V(z)|} .
$$

As argued in the proof of Theorem 3 for the function $\kappa_{d}$ defined in (A.3), we can assume without loss of generality that $\kappa_{c} \in \mathcal{K}_{\infty}$.

We start by picking arbitrary $x \in \mathbb{R}^{n}, w \in \mathbb{R}^{m}$ for which $\nabla V(x)$ exists and $z:=f(x, w)-f(x, 0)$ is "small" in the sense that it satisfies

$$
|z|=|f(x, w)-f(x, 0)|<\kappa_{c}(|x|) .
$$

Using the fact that $\nabla V(x) \cdot f(x, 0) \leq 0,($ A.9) and the definition of $\kappa_{c}$, we conclude that

$$
\begin{aligned}
\nabla V(x) & f(x, w)=\nabla V(x) \cdot f(x, 0)+\nabla V(x) \cdot z \\
& \leq \nabla V(x) \cdot z \leq|\nabla V(x)| \kappa_{c}(|x|) \leq-c V(x)
\end{aligned}
$$

Suppose that we now pick $x \in \mathbb{R}^{n}, w \in \mathbb{R}^{m}$ for which $\nabla V(x)$ exists and $z:=f(x, w)-f(x, 0)$ is "large" in the sense that it satisfies

$$
|x| \leq \kappa_{c}^{-1}(|z|)=\kappa_{c}^{-1}(|f(x, w)-f(x, 0)|)
$$

instead of (A.9). Since $V$ is locally Lipschitz, there exists a function $\alpha_{2} \in \mathcal{K}_{\infty}$ and a constant $k \geq 0$ such that $|\nabla V(x)| \leq k+\alpha_{2}(|x|) \forall x$ a.e. (cf. [6]). Because of this, the fact that $\nabla V(x) \cdot f(x, 0) \leq 0$, and (A.11), we now have

$$
\begin{gathered}
\nabla V(x) \cdot f(x, w)=\nabla V(x) \cdot f(x, 0)+\nabla V(x) \cdot z \\
\leq \nabla V(x) \cdot z \leq k|z|+\alpha_{2}(|x|)|z| \\
\leq k|z|+\alpha_{2} \circ \kappa_{c}^{-1}(|z|)|z| \leq \chi(|w|)
\end{gathered}
$$

with $\chi(s):=k \gamma(s)+\left(\alpha_{2} \circ \kappa_{c}^{-1} \circ \gamma\right)(s) \gamma(s) \forall s \geq 0$, where $\gamma$ is the growth estimate of $f(x, w)-f(x, 0)$. Combining (A.10) with (A.12), we conclude that (4a) holds for almost all $x$ and $w$, from which C5 follows.

C6. As in the proof of Theorem 3, one can show that C6 follows from C4 and C5.

\section{References}

[1] D. Angeli and E. Sontag. Forward completeness, unboundedness observability, and their Lyapunov characterizations. Syst. \& Contr. Lett., 38:209-217, 1999.

[2] D. Angeli, E. D. Sontag, and Y. Wang. A characterization of integral input-to-state stability. IEEE Trans. on Automat. Contr., 45(6):1082-1097, June 2000.

[3] D. D. Bainov and P. S. Simeonov. Systems with Impulse Effects: Stability, Theory and Applications. Academic Press, New York, 1989.

[4] C. Cai and A. R. Teel. Results on input-to-state stability for hybrid systems. In Proc. of the 44th Conf. on Decision and Contr., pages 5403-5408, Dec. 2005.

[5] C. Cai, A. R. Teel, and R. Goebel. Results on existence of smooth Lyapunov functions for (pre-)asymptotically stable hybrid systems with non-open basins of attraction. In Proc. of the 2007 Amer. Contr. Conf., pages 3456-3461, July 2007.

[6] F. H. Clarke. Optimization and Nonsmooth Analysis. SIAM, Philadelphia, PA, 1990.

[7] W. M. Haddad, V. S. Chellaboina, and S. G. Nersesov. Impulsive and Hybrid Dynamical Systems: Stability, Dissipativity, and Control. Princeton University Press, 2006.

[8] J. P. Hespanha. Stabilization through hybrid control. In H. Unbehauen, editor, Encyclopedia of Life Support Systems (EOLSS), volume Control Systems, Robotics, and Automation. Developed under the Auspices of the UNESCO, Eolss Publishers, Oxford, UK, 2004.

[9] J. P. Hespanha and A. S. Morse. Stability of switched systems with average dwell-time. In Proc. of the 38th Conf. on Decision and Contr., pages 2655-2660, Dec. 1999.

[10] J. P. Hespanha, A. Ortega, and L. Vasudevan. Towards the control of linear systems with minimum bit-rate. In Proc. of the Int. Symp. on the Mathematical Theory of Networks and Syst., Aug. 2002.

[11] J. P. Hespanha, D. Liberzon, and A. R. Teel. Lyapunov conditions for input-to-state stability of impulsive systems. Technical report, Univ. California, Santa Barbara, 2007. Available at http://www.ece.ucsb.edu/ hespanha/techrep.html.

[12] Z. P. Jiang and Y. Wang. Input-to-state stability for discrete-time nonlinear systems. Automatica, 37:857869, 2001.

[13] H. K. Khalil. Nonlinear Systems. Prentice-Hall, New Jersey, 3rd edition, 2002.

14] V. Lakshmikantham and S. Leela. Differential and Integral inequalities: Theory and Applications. Volume IOrdinary Differential Equations, volume 55 of Mathematics in Science and Engineering. Academic Press, New York, 1969.

[15] D. Liberzon. Switching in Systems and Control. Birkhäuser, Boston, 2003. 
[16] D. Liberzon and J. P. Hespanha. Stabilization of nonlinear systems with limited information feedback. IEEE Trans. on Automat. Contr., 50(6):910-915, June 2005.

[17] J. L. Mancilla-Aguilar and R. A. Garcia. On converse Lyapunov theorems for ISS and iISS switched nonlinear systems. Syst. \& Contr. Lett., 42:47-53, 2001.

[18] A. Mehta, S. Cherian, D. Hedden, and T. Thundat. Manipulation and controlled amplification of Brownian motion of microcantilever sensors. Applied Physics Letters, 78(11):1637-1639, Mar. 2001.

[19] D. Nesic and A. R. Teel. Input-output stability properties of networked control systems. IEEE Trans. on Automat. Contr., 49(10):1650-1667, Oct. 2004.

[20] S. Prajna, A. Papachristodoulou, P. Seiler, and P. A. Parrilo. SOSTOOLS: Sum of squares optimization toolbox for MATLAB, 2004.

[21] L. Praly and Y. Wang. Stabilization in spite of matched unmodeled dynamics and an equivalent definition of input-to-state stability. Math. Contr., Signals, Syst., 9: 1-33, 1996.

[22] E. D. Sontag. Comments on integral variants of ISS. Syst. \& Contr. Lett., 34:93-100, 1998.

[23] E. D. Sontag. Smooth stabilization implies coprime factorization. IEEE Trans. on Automat. Contr., 34(4): 435-443, Apr. 1989.

[24] E. D. Sontag and Y. Wang. On characterizations of the input-to-state stability property with respect to compact sets. In Proc. IFAC Non-linear Control Systems Design Symposium (NOLCOS'95), pages 226-231, June 1995.

[25] Y. Tao. Impulsive Control Theory, volume 272 of Lecture Notes in Control and Information Sciences. SpringerVerlag, Berlin, 2001.

[26] A. R. Teel and L. Praly. On assigning the derivative of a disturbance attenuation control Lyapunov function. Math. Contr., Signals, Syst., 13:95-124, 2000.

[27] G. C. Walsh, O. Beldiman, and L. G. Bushnell. Asymptotic behavior of nonlinear networked control systems. IEEE Trans. on Automat. Contr., 46(7):1093-1097, July 2001.

[28] Y. Xu and J. P. Hespanha. Communication logics for networked control systems. In Proc. of the 2004 Amer. Contr. Conf., pages 572-577, June 2004.

[29] W. Zhang and K. L. Turner. A mass sensor based on parametric resonance. In Proc. of the Solid-State Sensor, Actuator and Microsystems Workshop, Hilton Head Island, South Carolina, pages 49-52, June 2004.

[30] W. Zhang, R. Baskaran, and K. L. Turner. Effect of cubic nonlinearity on auto-parametrically amplified resonant MEMS mass sensor. Sensors and Actuators A, 102:139-150, 2002. 